Musées, Patrimoine et Culture scientifiques et techniques

$164 \mid 2016$

mars-avril 2016

\title{
Le profil socio-démographique et les activités des médiateurs de CSTI
}

\section{Stéphane Frugier}

\section{OpenEdition \\ Journals}

Édition électronique

URL : http://journals.openedition.org/ocim/1639

DOI : 10.4000/ocim. 1639

ISSN : 2108-646X

Éditeur

OCIM

Édition imprimée

Date de publication : 1 mars 2016

Pagination : $12-20$

ISSN : 0994-1908

Référence électronique

Stéphane Frugier, « Le profil socio-démographique et les activités des médiateurs de CSTI », La Lettre de I'OCIM [En ligne], 164 | 2016, mis en ligne le 01 mars 2017, consulté le 30 avril 2019. URL : http:// journals.openedition.org/ocim/1639; DOI : 10.4000/ocim.1639

Ce document a été généré automatiquement le 30 avril 2019

Tous droits réservés 


\section{Le profil socio-démographique et les activités des médiateurs de CSTI}

\section{Stéphane Frugier}

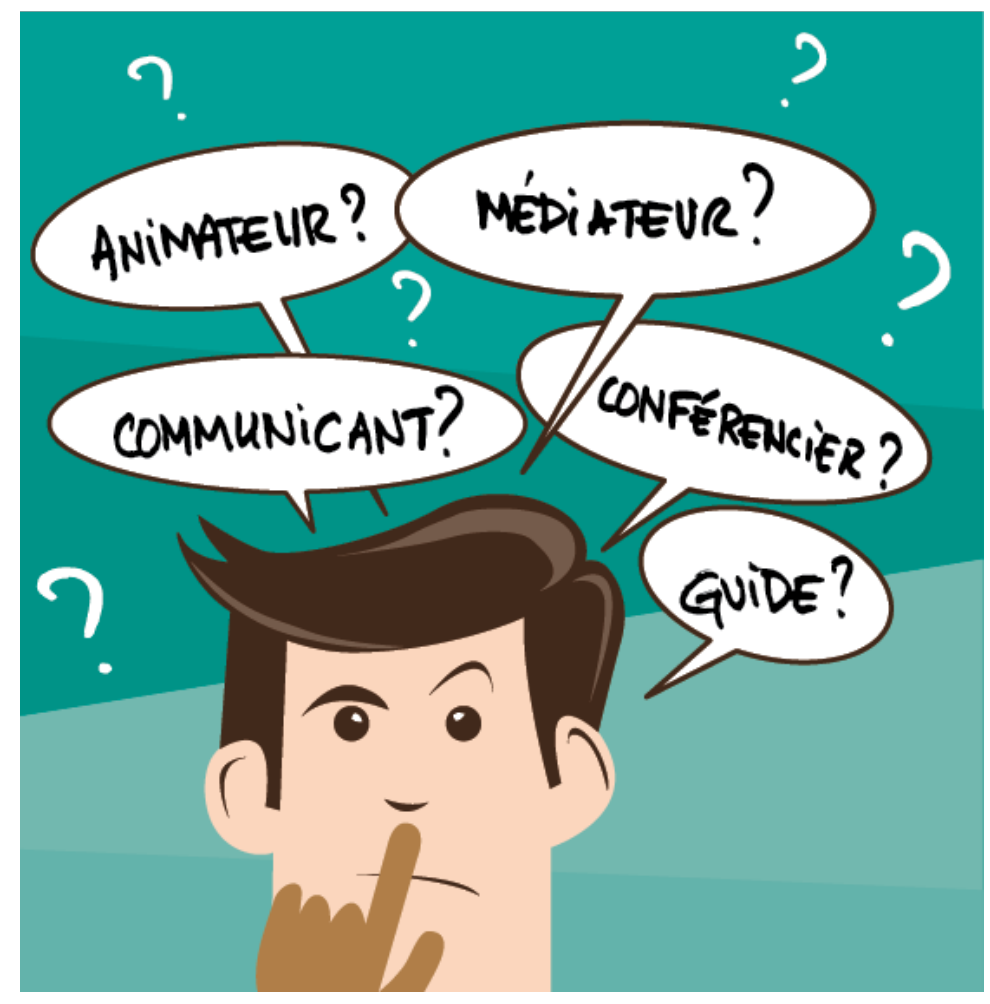

(c) Pierre Guillemeney/ibuc

1 Le pôle observation et veille du projet ESTIM École de la médiation a mené plusieurs enquêtes relatives aux médiateurs scientifiques (voir l'article précédent), dont une quantitative. Celle-ci, intitulée Les besoins en formation des médiateurs/animateurs scientifiques et de leurs encadrants, a été réalisée en 2013-2014 à l'aide de deux questionnaires (médiateurs et encadrants). Elle avait pour objectifs, entre autres : 
2 - de confirmer ou d'infirmer les connaissances actuelles (empiriques, qualitatives, subjectives) et les constats des enquêtes qualitatives du projet ;

3 - de cartographier les profils de ces acteurs en termes socio-démographiques, de type d'organisme dans lequel ils travaillent, de statut, d'activités ;

4 - de constituer de la matière pour la construction d'un référentiel métier;

5 - d'identifier les attentes et besoins en formation des médiateurs et de leurs encadrants (voir l'article précédent).

6 L'ensemble des résultats est disponible à travers une série de six documents: Méthodologie, Échantillon, Socio-démographie, L'intitulé de fonction, Les besoins de formation des médiateurs/animateurs scientifiques et Les activités des médiateurs/animateurs scientifiques. Ils sont accessibles sur le site Internet de l'École de la médiation, www.estim-mediation.fr ainsi que sur www.ocim.fr.

7 Outre une rapide description des caractéristiques de l'échantillon, les éléments rapportés ici constituent quelques résultats importants issus du questionnaire sur les médiateurs. Ils peuvent être présentés à travers deux interrogations relatives à une vision courante, sinon schématique, portée sur le métier de médiateur: l'assertion commune d'une profession féminine ne serait-elle pas à relativiser ? Par ailleurs, au-delà du foisonnement des intitulés de fonction et de la diversité des organismes, l'exercice de la fonction de médiateur peut-il être caractérisé par un socle commun d'activités propres à définir une profession?

8 L'échantillon, avec 431 réponses, a couvert le territoire français de manière relativement homogène (voir le document Échantillon). L'enquête était ouverte à toute personne exerçant une activité de médiateur, quel que soit l'intitulé de la fonction occupée ${ }^{1}$. De fait, sont représentés les organismes de CSTI (centres de sciences, muséums, musées et sites archéologiques/ethnologiques, musées techniques, musées de société) pour $65,9 \%$ des répondants, mais aussi les musées d'art et/ou d'histoire (10,2\%) les structures d'éducation populaire $(13,1 \%)$, les universités et organismes de recherche $(4,8 \%)$ et des structures diverses (6,1\% : écoles, office de tourismes, collectivités territoriales...).

9 Les participants ont aussi indiqué la fonction qu'ils occupent. Les intitulés de fonction sont répartis en trois catégories ${ }^{2}$ : la première réunit $74,3 \%$ d'entre eux avec les termes "médiateur" et "animateur"; la deuxième $(11,4 \%)$ comprend des termes liés à la médiation (chargés ou responsables d'animation/de médiation/des publics); la dernière $(14,3 \%)$ rassemble les autres intitulés de fonction (métiers du patrimoine ; des chargés de communication, de mission, de projet ; des guides...).

\section{Un univers plutôt féminin et très diplômé}

10 L'échantillon comprend $2 / 3$ de femmes $(66,1 \%)$. Ce résultat tend à confirmer celui de l'enquête Pilots (professionalisation in learning technology and science: projet soutenu par le programme européen à la formation continue, novembre 2008-octobre 2010, www.thepolits.eu) qui semblait montrer une surreprésentation des femmes parmi les médiateurs européens (l'échantillon Pilots comprenait 236 questionnaires: 2/3 de médiateurs, 1/3 de managers. "Il s'agit d'une profession majoritairement féminine: $61 \%$ des médiateurs des musées et centres de sciences européens sont des femmes. C'est strictement la même proportion que celle observée par Adam Love Rodgers et Bryony Kelly en 2001 dans les centres 
interactifs du Royaume Uni", Pilots, 2010). Toutefois, cette répartition femme/homme diffère selon le type d'organisme où les répondants exercent leurs fonctions : les hommes sont $43,6 \%$ en centres de sciences $(56,4 \%$ de femmes) et $50 \%$ dans les muséums et le patrimoine naturel (50\% de femmes), bien plus que dans l'Éducation populaire (30,2\% d'hommes, $69,8 \%$ de femmes) et surtout dans les autres musées, où ils sont au plus $20,2 \%$, soit 79,8\% de femmes (musée de CSTI, tel que musée et site archéologique/ethnologique, musée technique, musée de société).

\section{La formation initiale}

11 Alors que l'étude Pilots montrait une population fortement diplômée ("Le second phénomène remarquable est le haut niveau de diplôme des médiateurs interrogés : plus de la moitié d'entre eux détiennent un master ( $38 \%$ ) ou un doctorat (14\%)."), les études qualitatives menées dans le cadre de l'École de la médiation semblent plus nuancées, avec effectivement des professionnels pour certains très diplômés mais d'autres au contraire avec un bagage initial nettement moins important ${ }^{3}$. Le niveau de diplôme des répondants est particulièrement élevé : $63,6 \%$ ont un niveau égal ou supérieur à Bac+4. Les femmes sont un peu plus diplômées que les hommes, bien que cette différence soit assez faible : 67,4 \% des femmes ont Bac+4, contre 55,9\% des hommes. Le haut niveau de diplôme est en tout cas contradictoire avec $60 \%$ des fonctionnaires en catégorie $C$, pour laquelle le diplôme requis pour présenter un concours est seulement de niveau $\mathrm{V}$ (niveau $3^{\mathrm{e}}$, tels que CAP, $\mathrm{BEP})$.

12 L'étude européenne Pilots montrait que massivement les médiateurs venaient directement de cursus scientifiques ou technologiques - voire d'autres filières plus littéraires ou artistiques - mais qu'assez peu d'entre eux avaient suivi de formation initiale à la médiation. Les études qualitatives réalisées pour l'École de la médiation semblent également aller dans ce sens ${ }^{4}$. L'a-typicité des parcours initiaux des médiateurs semble bien être finalement la norme chez ces professionnels et une richesse plutôt qu'un handicap. Parmi les répondants, la discipline de formation initiale est très variée (voir Figure 1): les sciences de la vie et de la terre (14\%), archéologie, histoire de l'art (12\%) et développement durable, écologie, environnement (11\%) sont les plus représentés. Comme dans Pilots, les médiateurs issus de formation à la médiation sont peu nombreux, avec seulement 15,3\% de l'échantillon pour communication et médiation scientifiques et médiation et ingénierie culturelles. Ces dernières font par ailleurs souvent suite à un cursus plus "classique", que ce soit en sciences dites "exactes" (SVTU : sciences de la vie, de la terre et de l'univers ; maths, physique, chimie) ou humaines (archéologie, arts, histoire, histoire de l'art...). 
FIgURE 1 Discipline de formation initiale selon le type d'organisme

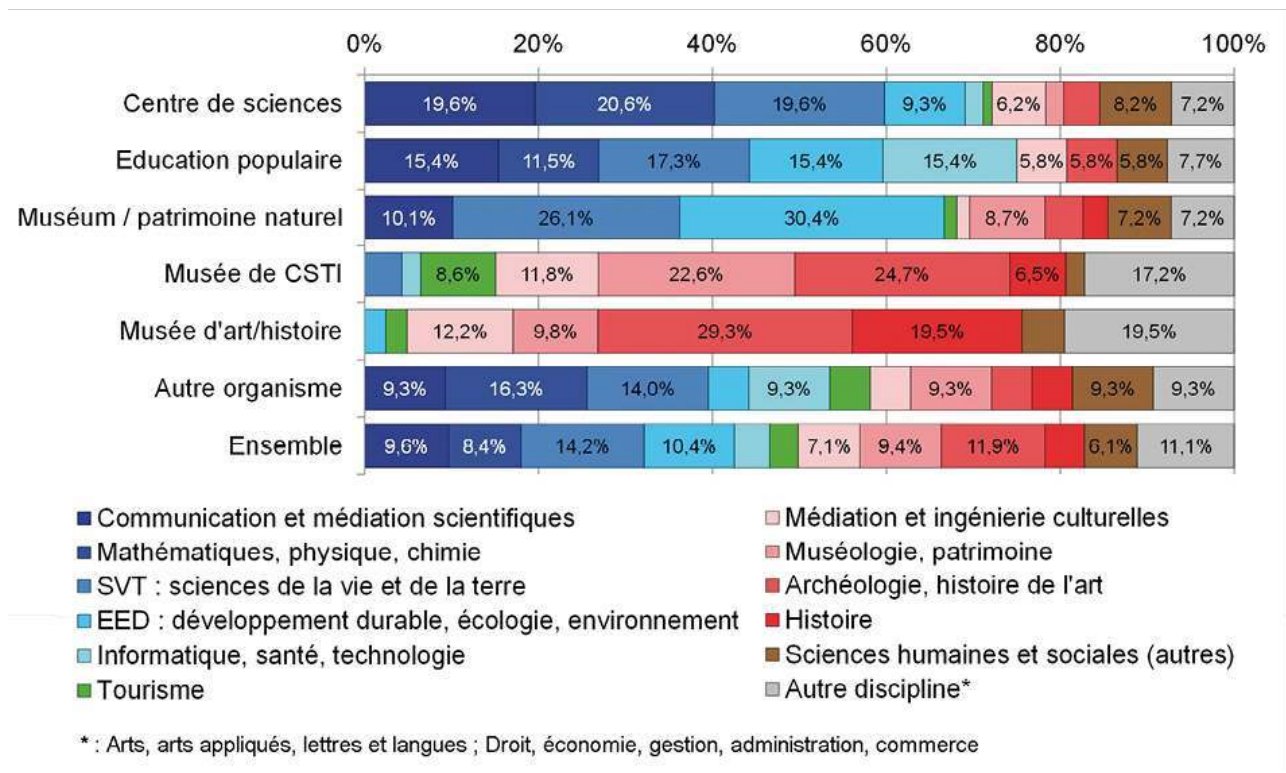

(c) OCIM

FIgURE 2 Discipline de formation initiale selon le sexe (en vert, les disciplines surreprésentées parmi les femmes et en violet, les disciplines surreprésentées parmi les hommes)

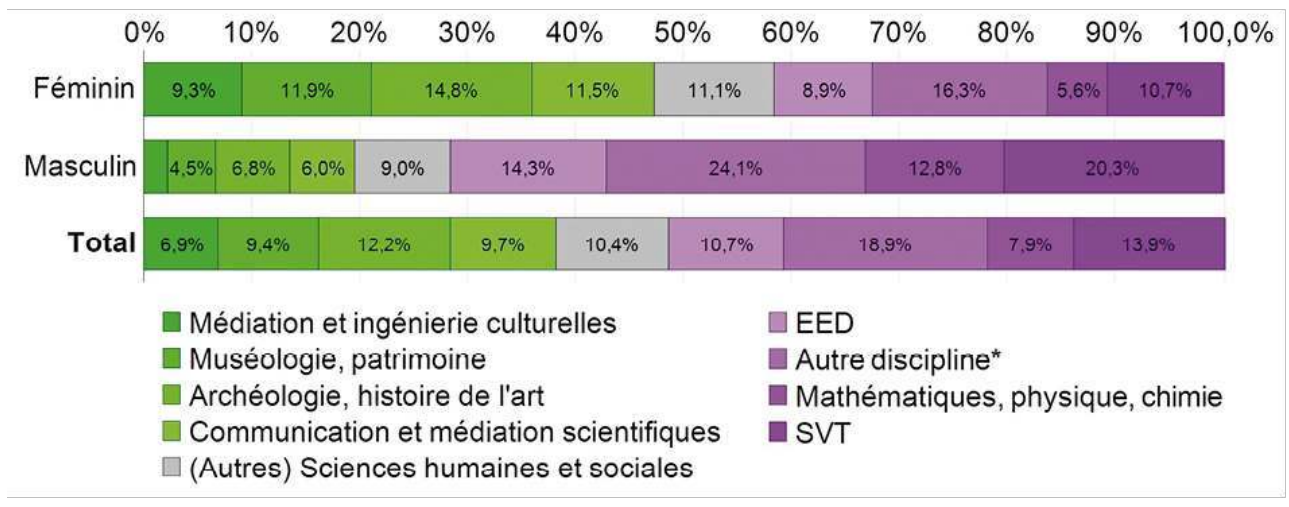

(C) OCIM 
FIgURE 3 Discipline de formation initiale selon l'âge (en vert, les disciplines surreprésentées parmi les répondants de moins de 35 ans et en violet, les disciplines surreprésentées parmi les répondants de 35 ans ou plus)

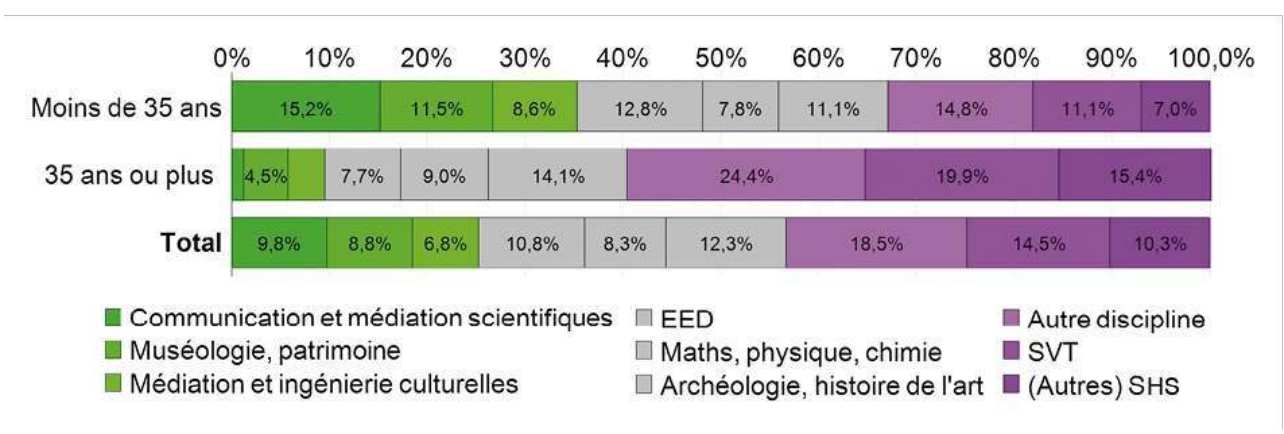

(C) OCIM

\section{Le type d'organisme}

\section{Générations}

15 Par ailleurs, les formations initiales à la médiation scientifique/culturelle sont relativement récentes : apparues dès le début des années 1990, l'offre est conséquente depuis une quinzaine d'années, notamment dans le cadre de la mise en place du schéma européen de l'enseignement supérieur LMD (licence-master-doctorat). Ainsi, les médiateurs de moins de 35 ans sont bien plus souvent issus des formations initiales à la médiation scientifique/culturelle et en muséologie/patrimoine que leurs ainés (respectivement $34,3 \%$ et 9,6\%, voir Figure 3). Or, ces formations sont aussi plus fréquentes parmi les femmes que parmi les hommes (respectivement $32,7 \%$ et $12,8 \%$, voir Figure 2), ce qui laisse supposer une féminisation encore accrue de la fonction dans les prochaines années. 


\section{Un métier aux multiples facettes}

\section{Âge, statut/contrat d'emploi et profession}

En attendant, l'âge recouvre une autre dimension. Les répondants ont 33,7 ans en moyenne, la moitié ont moins de 33 ans : ils sont donc plutôt jeunes (l'âge moyen des actifs est de 39,6 ans en 2010, in La population active en métropole à l'horizon 2030, Insee Première $n^{\circ} 1371$, octobre 2011). Pour autant, une part importante d'entre eux $(39,2 \%)$ a 35 ans ou plus, ce qu'illustre la structure par âge (voir Figure ci-dessus) et correspond assez peu à l'hypothèse selon laquelle la fonction de médiateur ne constituerait pas une profession mais un passage transitoire avant d'occuper un poste "réel".

Parallèlement, en termes de statuts/contrats d'emploi, une situation pérenne (CDI et statut de fonctionnaire) concerne plus de la moitié des répondants $(57 \%)$ alors que les vacataires (cette catégorie comprend les vacataires, les CDD de moins de $8 \mathrm{~h} / \mathrm{semaine}$, les conventions de prestation et l'auto-entreprenariat) n'en constituent qu'à peine $12 \%$ et les CDD $27 \%$ (bénévoles et stagiaires forment le reste, moins de $4 \%$ ). Il est très probable que ces vacataires soient sous-représentés, en raison d'une part de la difficulté à les informer (cf. document Méthodologie de l'enquête), d'autre part du risque qu'ils ne se soient pas sentis suffisamment concernés par le questionnaire, précisément du fait de leur situation : activité récente et/ou jugée temporaire, à l'image des étudiants ou de jeunes diplômés.

Bien que l'échantillon ne reflète de la réalité qu'une part réduite des acteurs temporaires, la présence de médiateurs en situation pérenne et, de surcroit, âgés de 30 ans ou plus, est importante : elle représente près de la moitié $(46 \%)$ de l'échantillon. De fait, pour une part des répondants non marginale, les caractéristiques de l'échantillon en termes d'âge et de statuts/contrats d'emploi tendent nettement vers une fonction occupée par des professionnels exerçant un (des) métier(s), dont les activités étaient par ailleurs questionnées. 


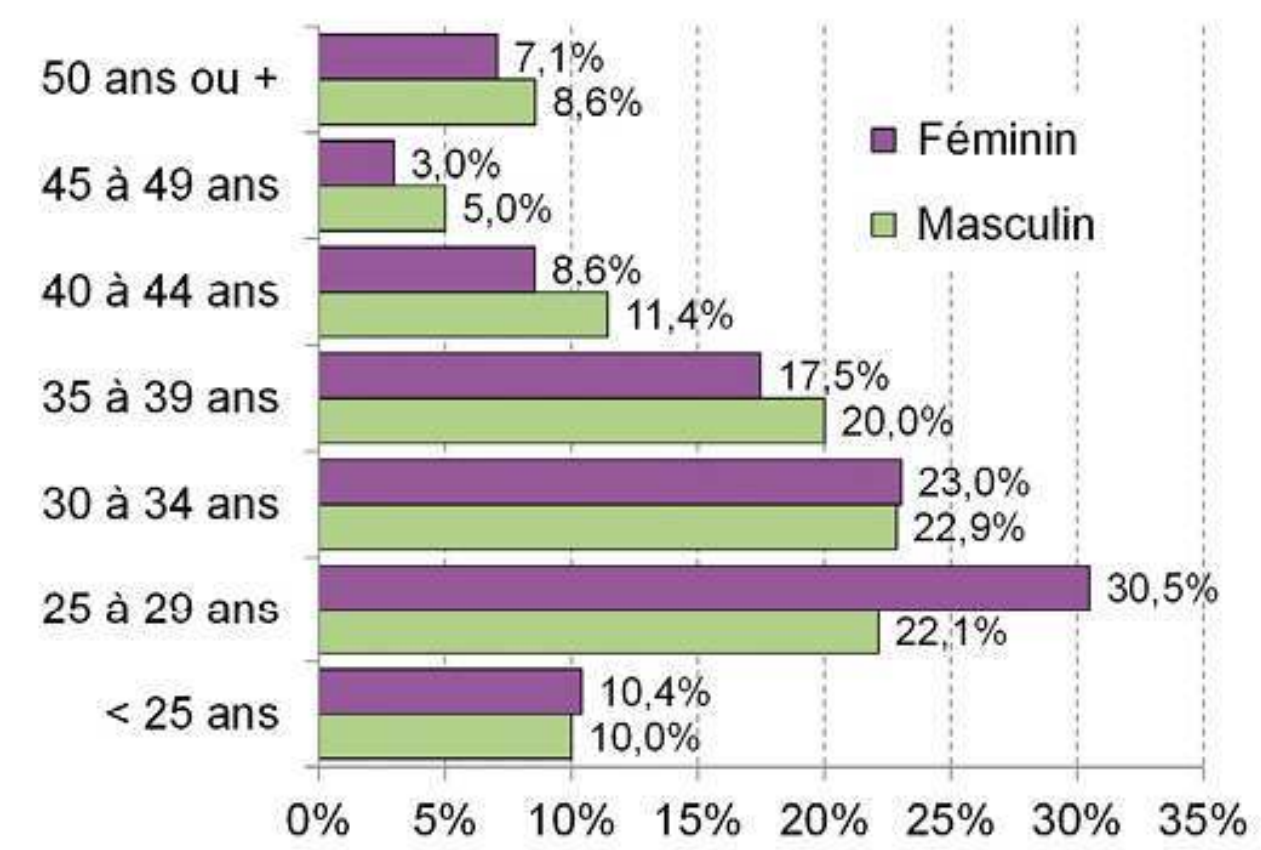

(C) OCIM

\section{Une large palette d'activités} importance relative: certaines activités sont moins fréquentes mais aussi plus souvent secondaires, telles la formation par des pairs et la conférence (la formation par des pairs est une activité principale pour $9 \%$ des répondants et une activité secondaire pour $29 \%$; pour la conférence, les résultats sont respectivement de $9 \%$ et $24 \%$ ) ; de plus, à fréquence similaire, certaines activités sont plus importantes que d'autres, comme l'est la conception de programme par rapport à la veille/actualité scientifique (ces activités sont toutes deux 
pratiquées par $50 \%$ des répondants ; la première est une activité principale pour $28 \%$ des répondants, alors que la seconde atteint seulement $18 \%$ ).

\section{Un cœur de métier, mâtiné de diversité}

Le cœur de la fonction de médiateur, quel que soit l'intitulé, peut être défini par les activités les plus partagées et importantes, par exemple par plus de $60 \%$ des répondants (voir Figure 4) et dont la majorité les exercent en activité principale : Animation d'ateliers [...], Conception d'ateliers [...], Réalisation d'outils pédagogiques [...], Visite guidée, Participation à la réalisation d'outils de médiation [...], Organisation d'événements/gestion de projet, Autoformation sur contenus scientifiques [...]. Le cœur de cette fonction englobe donc plusieurs activités qui dépassent le seul cadre de l'interaction avec le public. De plus, si la conférence est la moins pratiquée des activités proposées, elle l'est tout de même par $1 / 3$ des répondants. L'exercice de la fonction de médiateur comprend donc une large palette d'activités différentes.

Parallèlement, un même médiateur a bien une activité diversifiée : le nombre de types d'activités pratiquées est important (11,2 en moyenne; près de $2 / 3$ des médiateurs en déclarent entre 7 et 15 ; seuls $4,0 \%$ pratiquent moins de 4 activités différentes). Le nombre moyen d'activités principales $(5,9)$ est légèrement supérieur à celui des activités secondaires $(5,3)$, soit un peu plus d'activités principales dans l'ensemble des activités pratiquées (55\%). Toutefois, les activités principales représentent plus de $2 / 3$ des activités pratiquées pour $25 \%$ des médiateurs et, à l'opposé, elles en constituent moins de $1 / 3$ pour $15 \%$ d'entre eux. Ainsi, le nombre d'activités différentes pratiquées par un même médiateur est globalement important bien que variable et la part d'activités principales varie assez fortement d'un individu à l'autre.

FIgURE 4 Types d'activités pratiquées par les médiateurs (la ligne rouge délimite les activités pratiquées par au moins $60 \%$ des répondants).

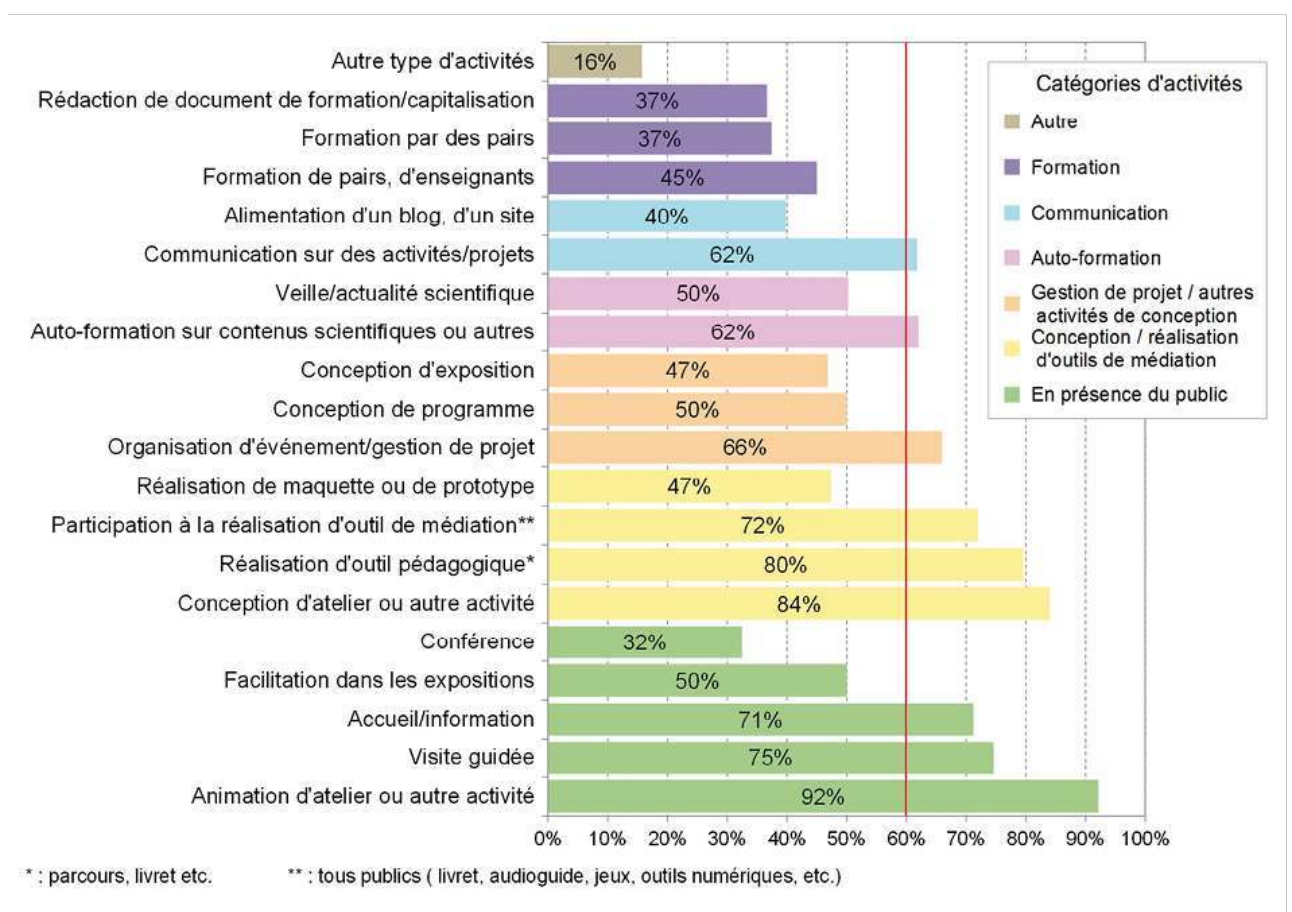

(C) OCIM 
FIgURE 5 Pratique d'activité(s) par catégorie : parmi les activités de la catégorie "en présence du public", 91 \% des répondants en pratiquent une ou plusieurs comme activité principale, moins de $8 \%$ en pratiquent (une ou plusieurs) uniquement comme activité secondaire et $2 \%$ n'en pratiquent aucune.

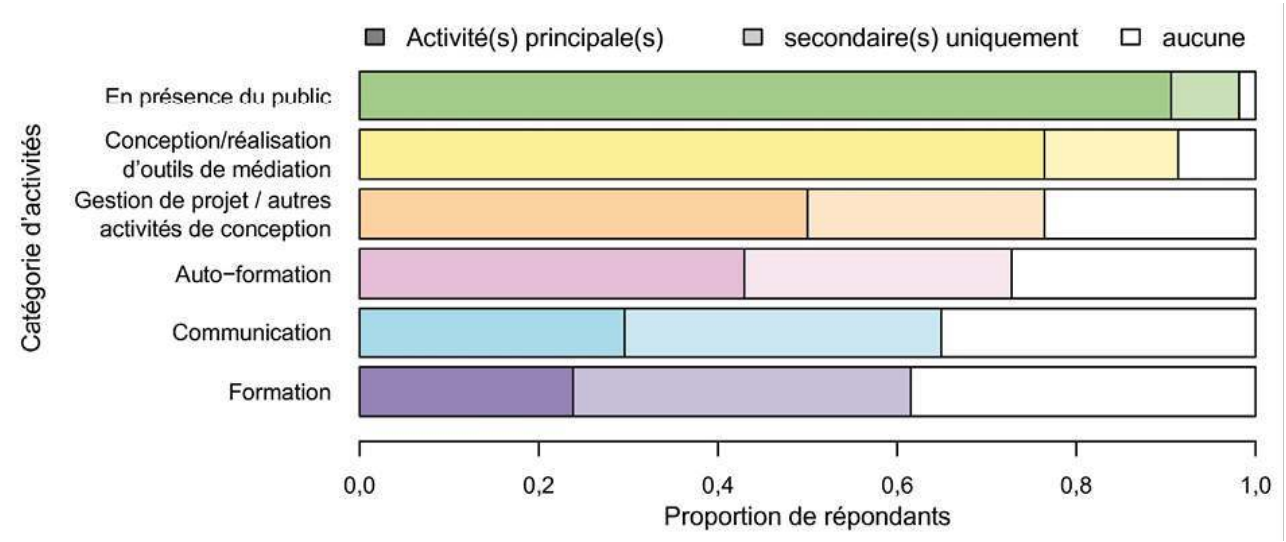

(C) OCIM

\section{Des activités hiérarchisées}

27 Les activités ont été analysées après un travail de catégorisation. Cette dernière permet de regrouper des types d'activités dont l'exercice différe d'un organisme à l'autre, par l'importance donnée, les objectifs assignés et les formes pratiquées.

En ce sens, les activités En présence du public et de Conception / réalisation d'outils de médiation sont les plus répandues et importantes (voir Figure 4 et Figure 5), alors que celles de Communication et de Formation sont bien plus secondaires.

La répartition par catégorie du nombre moyen d'activités pratiquées (voir Figure 6) illustre la place majeure que constituent les activités autres que celles en présence du public, relativisant l'importance de ces dernières dans l'exercice de la fonction de médiateur. Les catégories En présence du public et Conception / réalisation d'outils de médiation forment 2/3 des activités principales (voir Figure 7), bien qu'elles ne constituent que la moitié des activités proposées (9 sur 19). Les catégories Gestion de projet / autres activités de conception et Auto-formation regroupent $1 / 5$ des activités (1/4 de celles proposées) et celles de Communication et Formation n'en réunissent que $1 / 10$ (1/4 de celles proposées).

30 Ainsi, la fréquence et l'importance des activités hiérarchisent les catégories :

31 - des activités de médiation essentielles : En présence du public et Conception / réalisation d'outils de médiation;

32 - des activités de médiation complémentaires: Gestion de projet / autres activités de conception et Auto-formation ;

з3 - des activités périphériques : Communication et Formation.

\section{De l'intitulé de fonction}

L'importance relative des catégories d'activités principales et secondaires est liée à certaines caractéristiques des médiateurs. En effet, les animateurs/médiateurs et chargés/ 
responsables de $\mathrm{A} / \mathrm{M} / \mathrm{P}$ (animation/médiation/publics) déclarent, en moyenne, un nombre d'activités principales plus important que les autres intitulés (respectivement 6,$2 ; 6,8$ et 3,9 activités).

Parallèlement, les activités de médiation essentielles forment, en moyenne, $70 \%$ des activités des animateurs/médiateurs, $60 \%$ de celles des chargés/responsables de A/M/P et $40 \%$ pour les autres intitulés. Les activités en présence du public sont plus importantes pour les animateurs/médiateurs que pour ceux ayant d'autres intitulés de fonction (voir Figure 8). De même, la conception/réalisation d'outils de médiation est bien plus pratiquée par les acteurs dont l'intitulé de fonction est relatif à la médiation (animateur/médiateur et chargé/resp. de A/M/P) que par les autres intitulés. Les animateurs/médiateurs ont aussi une pratique plus répandue d'auto-formation alors que les activités de gestion de projet et autres activités de conception sont plus fréquentes parmi les chargés/resp. de $\mathrm{A} / \mathrm{M} / \mathrm{P}$. Par contre, les activités périphériques constituent à peine $10 \%$ des activités des animateurs/ médiateurs, $17 \%$ de celles des chargés/resp. de A/M/P et $30 \%$ pour les autres intitulés. La communication et les autres activités sont moins répandues parmi les animateurs/médiateurs et tiennent une place importante pour les autres intitulés.

\section{Médiateur ou animateur?}

L'utilisation des intitulés animateur et médiateur pose la question de leur distinction ${ }^{6}$. La répartition des catégories d'activités principales semble légèrement différente entre médiateurs et animateurs : les activités en présence du public occuperaient une part un peu plus importante pour les animateurs et inversement pour celles de gestion de projet [...]. C'est en fait selon le type d'organisme que ces deux intitulés de fonction n'ont pas la même répartition d'activités. Dans les centres de sciences, la part des activités principales en présence du public est bien plus faible pour les médiateurs (34\%) que pour les animateurs (49\%) ; par contre, cette part est similaire dans les structures muséales/patrimoniales, quel que soit l'intitulé (respectivement, $42 \%$ et $38 \%$ ). Cette différence se retrouve à propos des activités de gestion de projet [...] et de communication. Il semblerait que l'absence de référence commune d'une définition distincte des fonctions d'animateur et de médiateur (culturels) se traduise par l'utilisation de l'un ou l'autre de ces intitulés pour des activités parfois identiques, parfois différentes, en fonction d'autres déterminants (type d'organisme, dimension muséale...). 
FIgURE 6 Nombre moyen d'activités principales et secondaires pratiquées par catégorie : les répondants pratiquent en moyenne 11,2 activités principales et secondaires, dont près de 2 activités principales en présence du public.

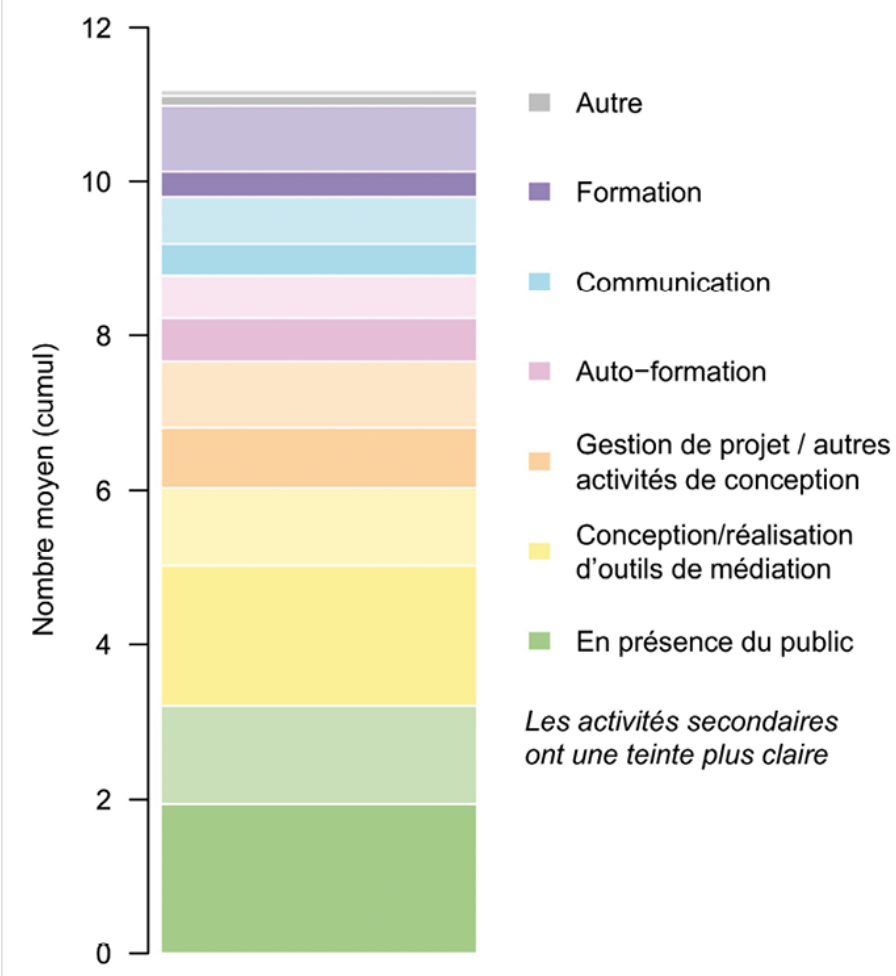

FIgURE 7 Nombre moyen d'activités principales pratiquées par catégorie : les répondants pratiquent 5,9 activités principales en moyenne, dont 1,9 en présence du public. Ainsi, en moyenne, $66 \%$ des activités principales pratiquées sont des activités de médiation essentielles.

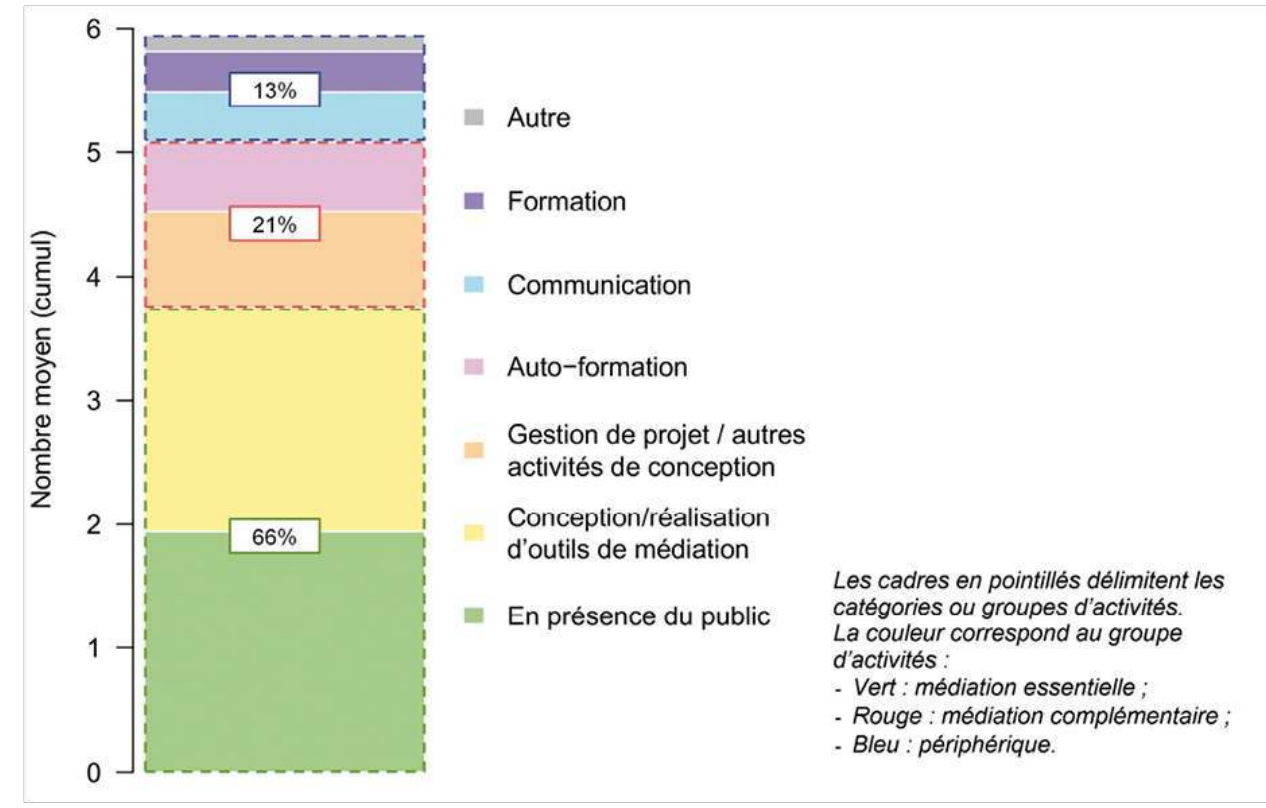

\section{(C) OCIM}


FIgURE 8 Proportion moyenne du nombre d'activités principales par catégorie selon l'intitulé de fonction : parmi les activités principales pratiquées, la part des activités de communication est, en moyenne, moins importante parmi les animateurs/médiateurs que parmi les chargés/resp. de A/M/ $P$, elle-même moins importante que parmi les autres intitulés de fonction.

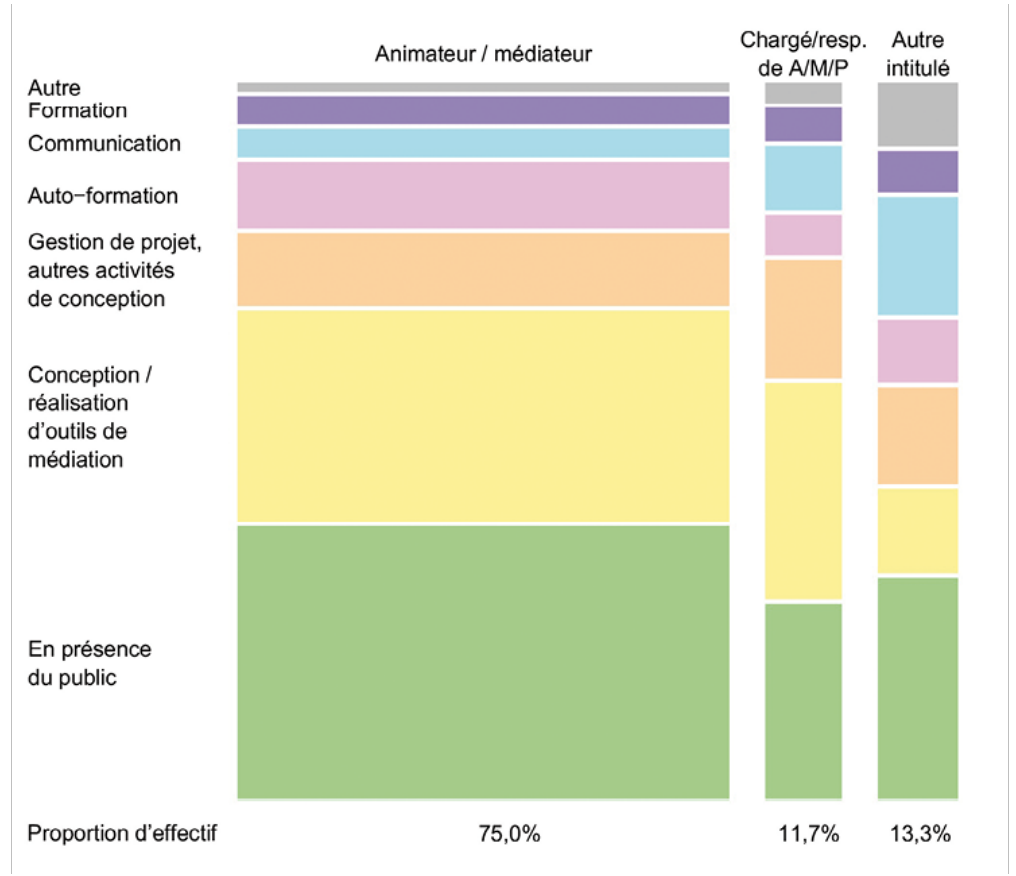

FIgURE 9 Proportion moyenne du nombre d'activités principales par catégorie selon le profil

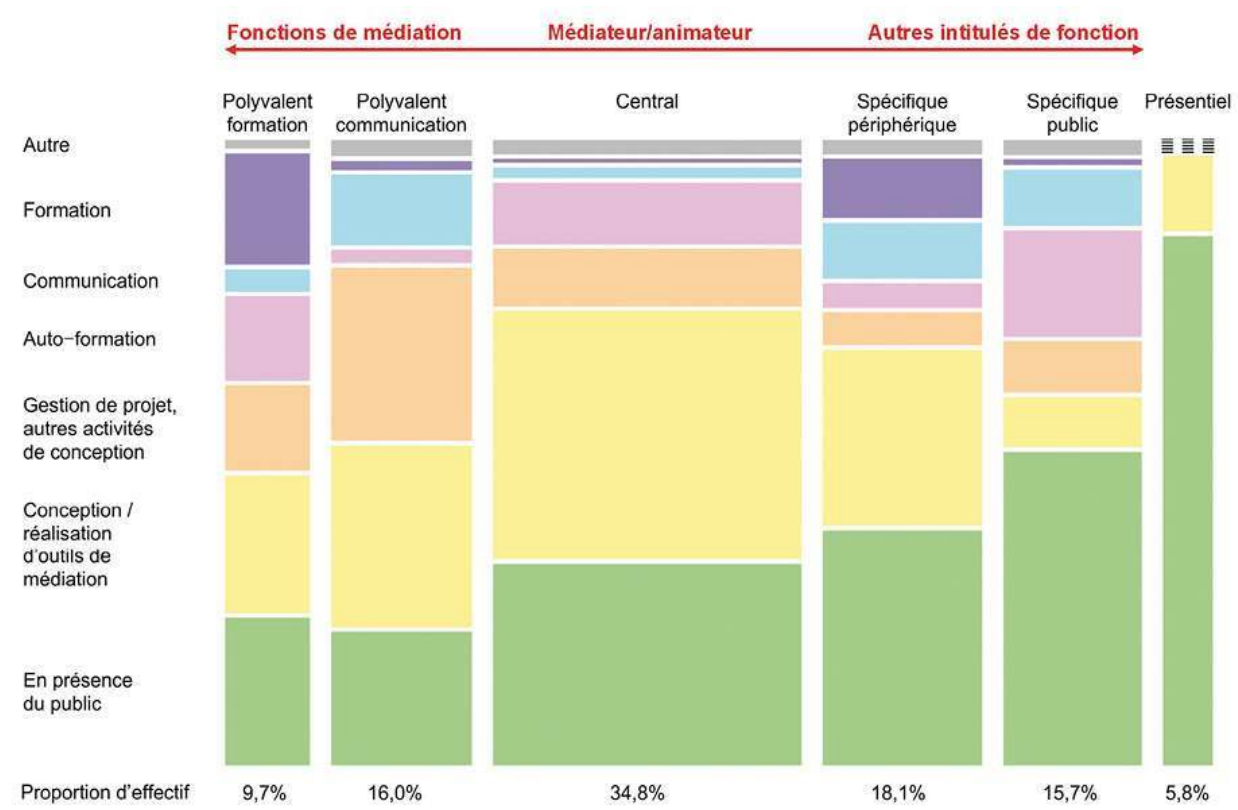

(c) OCIM 


\section{Des profils de médiateurs}

37 Le type d'organisme n'a qu'un faible lien avec la répartition des catégories d'activités pratiquées - si ce n'est marginal, en écartant les organismes les plus éloignés du champ culturel (c'est-à-dire les autres organismes: universités et organismes de recherche, collectivités territoriales et offices de tourisme, établissements scolaires...). Par contre, le niveau de diplôme, le statut/contrat d'emploi et l'intitulé de fonction ont un lien un peu plus prononcé avec cette répartition. Par ailleurs, bien d'autres facteurs peuvent être déterminants, comme l'organisation de la division du travail au sein des organismes, les formes de médiation qu'ils peuvent privilégier, ou bien encore les trajectoires professionnelles individuelles des médiateurs. Ainsi, la répartition des catégories d'activités n'apparait pas la même pour tous et peut se poser la question de savoir si, par exemple, certains acteurs ne seraient pas essentiellement en présence du public pendant que d'autres effectueraient surtout de la gestion de projet et/ou d'autres activités de conception?

38 L'hypothèse de l'existence de groupes d'acteurs exerçant certaines catégories d'activités de manière plus ou moins prononcée, est testée à partir de la répartition par catégorie du nombre moyen d'activités pratiquées (présentée Figure 6). Effectivement, divers profils de médiateurs se dégagent de la structuration générale. Le plus répandu, profil central $(34,8 \%)$, est avant tout constitué d'activités de médiation essentielles ( $3 / 4$ des activités principales), puis d'activités de médiation complémentaires $(1 / 5$ des activités principales). Les activités de gestion de projet et autres activités de conception sont le plus souvent des activités secondaires, celles périphériques à la médiation le sont presque exclusivement. De ce profil s'en éloignent d'autres (voir Figure 9), progressivement, soit par une plus grande polyvalence (profils polyvalents), soit par la prédominance des activités présentielles et une diminution de la quotité de travail en tant que médiateur (profils spécifiques et présentiel). Dans les deux cas, la conception/réalisation d'outils de médiation occupe une place de plus en plus restreinte.

\section{Un métier comme un autre}

Dans le même temps, s'écarter du profil central s'accompagne aussi d'un éloignement des (intitulés de) fonctions médiateur et animateur - ces deux intitulés ne présentant pas de différence notable en termes d'activités pratiquées - soit vers des intitulés relatifs à la médiation (profils polyvalents), soit vers d'autres intitulés de fonction (profils spécifiques). Exception d'importance, l'éloignement du profil présentiel par rapport au profil central en termes d'activités pratiquées mais aussi de quotité de travail et de type de contrat - ne s'accompagne pas d'une augmentation des autres intitulés de fonction (ce profil n'en comprend même aucun), ce qui pose la question de la distinction, en termes d'intitulés, entre cette fonction particulière (profil présentiel) occupée par des animateurs/médiateurs et celle occupée par les acteurs du profil central, essentiellement animateurs/médiateurs (ils constituent $86 \%$ du profil central).

Ces éloignements conjoints n'ont toutefois rien d'exceptionnel et s'inscrivent d'abord dans la diversité des pratiques d'un métier, ensuite dans le glissement progressif de l'exercice d'un métier vers celui d'autres métiers ou, autrement dit, dans l'exercice 
d'autres métiers comportant des activités faisant appel à des compétences communes voire propres - au premier.

Malgré la variété des conditions et modalités d'exercice de ce métier de médiateur/ animateur culturel (l'importance, les objectifs et les formes de la médiation sont propres à chaque organisme, notamment en fonction de son domaine et de ses missions), les différents profils d'activité existent dans tous les types d'organismes et ce dans des proportions similaires ou peu différentes. Par contre, ce n'est pas le cas des intitulés de fonction médiateur et animateur, ni de leurs qualificatifs scientifique ou culturel (cf. document Intitulé de fonction, partie I.3. L'intitulé de fonction et le type d'organisme). Cette absence d'unicité dans la dénomination de fonctions exerçant les mêmes types d'activités est donc fortement liée au type d'organisme, comme le reflet d'un "métier en quête d'identité"7, c'est-à-dire d'un manque de reconnaissance de ce métier dont les compétences (spécifiques pour certaines), lisibles ici dans les activités pratiquées et leur importance relative, forment pourtant dans leur association sa particularité et son identité.

\section{NOTES}

1. Pour plus de précisions sur l'échantillonnage, voir Méthodologie de l'enquête.

2. 135 intitulés de fonction différents ont fait l'objet d'une catégorisation qui peut être interprétée selon la proximité a priori avec la fonction de médiateur, voir le document L'intitulé de la fonction.

3. Belaën, F. et Mathieu, A.-L. Besoins en formation des médiateurs : étude qualitative dans des institutions patrimoniales de Bourgogne/Franche-Comté. 2013.

4. Garattini, L. Synthèse de quatre études qualitatives menées auprès des acteurs de la médiation (médiateurs et encadrants) sur les profils et les besoins en formation. Université Paris-Diderot, www.estim-mediation.fr/ressource/nthese-etudes-qualitatives-besoins-formation.

5. 382 répondants (soit $89 \%$ de l'échantillon) ont rempli cette partie du questionnaire. La répartition des répondants à la partie "Activités" selon le type d'organisme dans lequel ils travaillent ou selon leur intitulé de fonction n'est pas différente de celle de l'ensemble des répondants.

6. Voir notamment Peyrin, A. La question du nom, in Être médiateur au musée - Sociologie d'un métier en trompe-l'œil. 2010.

7. Peyrin, A. Être médiateur au musée - Sociologie d'un métier en trompe-l'œil. 2010 


\section{RÉSUMÉS}

Cette contribution présente les principaux résultats et les premières analyses d'une enquête quantitative menée dans le cadre de l'École de la médiation : une probable féminisation accrue de la fonction de médiateur dans les prochaines années, une diversité des pratiques et une absence d'unicité dans la dénomination de la fonction contribuant à un manque de reconnaissance du métier.

INDEX

Mots-clés : médiateur, csti, médiation, estim

\section{AUTEUR}

\section{STÉPHANE FRUGIER}

chargé des études quantitatives à l'OCIM, Observatoire du patrimoine et de la culture scientifiques et techniques

stephane.frugier@u-bourgogne.fr 